\title{
Structural understanding of the transport cycle of glutamate transporters
}

\author{
Xiaoyu Wang ${ }^{1, *}$ and SeCheol $\mathrm{Oh}^{2}$ \\ ${ }^{1}$ Department of Physiology and Biophysics, Weill Cornell Medicine, New York, NY 10065, USA \\ ${ }^{2}$ Structural Biology Program, Memorial Sloan Kettering Cancer Center, New York, NY 10065, USA \\ ${ }^{*}$ Correspondence: xiw2013@med.cornell.edu
}

Since the determination of the first crystal structure of a glutamate transporter homolog $\mathrm{Glt}_{\mathrm{ph}} 16$ years ago, the structural biology of glutamate transporter has progressed remarkably. To understand the complicated transport cycle of ion-coupled glutamate transport, each state's high-resolution structural information in the transport cycle is essential. Many of the structural information and insights about the sodium-coupled symport mechanism were gained from structural studies of prokaryotic homologs sodium-aspartate symporters, $\mathrm{Glt}_{\mathrm{Ph}}$ and $\mathrm{Glt}_{\mathrm{Tk}}$. Moreover, further insights were gained from recently unveiled structures of mammalian glutamate transporters (EAATs). Here we review $\mathrm{Glt}_{\mathrm{ph}}$, $\mathrm{Glt}_{\mathrm{T}}$, and mammalian glutamate transporters' structures and discuss how they help us to understand the ion-coupled transport mechanism in glutamate transporters.

\section{INTRODUCTION}

Glutamate is the primary excitatory neurotransmitter in the central nervous system (CNS). Following glutamate release into the synaptic cleft, extracellular glutamate is rapidly removed by plasma membrane-bound glutamate transporters to maintain normal neuron function and prevent excitotoxicity. In human, there are five glutamate transporter homologs named excitatory amino acid transporters (EAAT) 1-5. EAATs are not only found in the CNS, but also are expressed in other organs such as the kidney (EAAT3) (Danbolt, 2001). EAAT5 is exclusively expressed in the retina, where its glutamate activated large chloride conductance is essential for the light response (Veruki et al., 2006). EAATs belong to the solute carrier 1 A (SLC1A) family. They are secondary active transporters that couple the active uptake of glutamate to the co-transport of three sodium $\left(\mathrm{Na}^{+}\right)$ions and one proton $\left(\mathrm{H}^{+}\right)$, and the counter-transport of one potassium $\left(\mathrm{K}^{+}\right)$ ion (Zerangue and Kavanaugh, 1996). Besides L-glutamate, the transporter could also transport L- or D-aspartate (Menaker et al., 2006). In addition to the coupled transport, a thermodynamically uncoupled chloride $\left(\mathrm{Cl}^{-}\right)$conductance is activated by the binding of $\mathrm{Na}^{+}$ions and substrate (Fairman et al., 1995).

During the past two decades, with the availability of crystal and cryo-EM structures of glutamate transporter homologs in various states, understanding of the molecular mechanisms of glutamate transporters has grown dramatically. A vast majority of the structures available were acquired from two archaeal homologs of EAATs, Glt $\mathrm{Ph}_{\mathrm{Ph}}$ and $\mathrm{Glt}_{\mathrm{Tk}}$ (Yernool et al., 2004; Boudker et al., 2007; Reyes et al., 2009; Verdon and Boudker, 2012; Jensen et al., 2013; Verdon et al., 2014; Akyuz et al., 2015; Guskov et al., 2016; Scopelliti et al., 2018; Arkhipova et al., 2020; Huang et al., 2020; Wang and Boudker, 2020). Like EAATs, Glt $t_{p h}$ and $\mathrm{Glt}_{\mathrm{TK}}$ symports three $\mathrm{Na}^{+}$ions coupled with one L-aspartate (Asp) across the membrane, and an uncoupled anion conductance also has been reported in Glt $t_{\mathrm{Ph}}$ (Ryan and Mindell, 2007; Groeneveld and Slotboom, 2010). In contrast to EAATs, they do not require $\mathrm{H}^{+}$-symport or $\mathrm{K}^{+}$-antiport to complete the transport cycle. Since the first structure of Glt $t_{p h}$ was solved in 2004, Glt and $\mathrm{Glt}_{\mathrm{Tk}}$ have been used as excellent model systems to study glutamate transporters. Recently, structural studies of the human variants have extended the field and have been mostly consistent with earlier findings on Glt $\mathrm{Ph}_{\mathrm{Ph}}$ and $\mathrm{Glt}_{\mathrm{TK}}$ (Canul-Tec et al., 2017). In this review, we describe published structures of glutamate transporter homologs. Together, these studies suggest what appears to be a complete picture of the structural changes that underlie the ion-coupled substrate transport.

\section{STRUCTURE OF GLUTAMATE TRANSPORTER HOMOLOG GLT $_{\text {PH }}$}

The first structure of glutamate transporter was solved in 2004 from Glt $_{\mathrm{Ph}}$, an archaeal homolog of EAATs from Pyrococcus horikoshii (Yernool et al., 2004). Glt $\mathrm{t}_{\mathrm{Ph}}$ shares approximately $35 \%$ amino acid sequence identity to the EAATs. The Glt crystal structure shows that the transporter is a bowl-shaped homo-trimer with a solvent-filled extracellular basin extending halfway across the membrane bilayer. The trimer comprises a centrally located scaffold or trimerization domain and three peripheral transport domains that harbor independent substrate translocation pathways (Figure 1A). In each subunit, the scaffold domain includes TM 1, 2, 4, 5 and mediates all inter-protomer interactions. The transport domain consists of $\mathrm{TM} 3,6,7,8$, and two helical hairpins (HPs): an intracellular HP1 and an extracellular HP2. The transport domain harbors Asp (Figure 1B). The residues involved in substrate coordination are mostly at HP2 and TM8 (Figure 3A). In this structure, the substrate is 
TABLE 1 | Published structures and information of the glutamate transporters Glt $_{\mathrm{ph}}$, Glt $\mathrm{T}_{\mathrm{Tk}}$ and EAAT

\begin{tabular}{|c|c|c|c|c|c|c|c|c|}
\hline Protein & State & Ligand & $\begin{array}{l}\text { PDB } \\
\text { code }\end{array}$ & EMDB id & $\begin{array}{l}\text { Structure } \\
\text { determination } \\
\text { method }\end{array}$ & $\begin{array}{l}\text { Resolu- } \\
\text { tion }(\AA \AA)\end{array}$ & $\begin{array}{l}\text { Year of } \\
\text { release }\end{array}$ & Reference \\
\hline $\mathrm{Glt}_{\mathrm{Ph}}$ & OFS & & $1 \mathrm{XFH}$ & & X-ray diffraction & 3.5 & 2004 & DOI: 10.1038/nature05455 \\
\hline Glt $_{\mathrm{Ph}}$ & OFS-Asp & Asp & $2 \mathrm{NWL}$ & & X-ray diffraction & 2.96 & 2007 & DOI: 10.1038/nature05455 \\
\hline Glt $_{\text {ph }}$ & OFS-Asp & $\begin{array}{l}\text { Asp } \\
\mathrm{Na}\end{array}$ & $2 N W X$ & & X-ray diffraction & 3.29 & 2007 & DOI: 10.1038/nature05455 \\
\hline Glt $_{\mathrm{ph}}$ & OFS-TBOA & TBOA & 2NWW & & X-ray diffraction & 3.20 & 2007 & DOI: 10.1038/nature05455 \\
\hline $\begin{array}{l}\text { Glt } \\
\text { K55C/A364C }\end{array}$ & IFS-Asp & $\begin{array}{l}\text { Asp } \\
\mathrm{Na}\end{array}$ & $3 \mathrm{KBC}$ & & X-ray diffraction & 3.51 & 2009 & DOI: 10.1038/nature08616 \\
\hline $\begin{array}{l}\text { Glt }_{\text {ph }} \\
\text { V216C/M385C }\end{array}$ & IFS-Asp & $\begin{array}{l}\text { Asp } \\
\mathrm{Na}\end{array}$ & $3 \mathrm{~V} 8 \mathrm{~F}$ & & X-ray diffraction & 3.80 & 2012 & DOI: $10.1038 / n s m b .2233$ \\
\hline $\begin{array}{l}\text { Glt }_{\text {ph }} \\
\text { V198C/A380C }\end{array}$ & $\begin{array}{l}2 \text { IFS-Asp } \\
1 \text { iOFS-Asp }\end{array}$ & $\begin{array}{l}\text { Asp } \\
\mathrm{Na}\end{array}$ & $3 V 8 G$ & & X-ray diffraction & 4.66 & 2012 & DOI: 10.1038/nsmb.2233 \\
\hline $\begin{array}{l}\mathrm{Glt}_{\mathrm{ph}} \\
\mathrm{L66C/} \\
\mathrm{S} 300 \mathrm{C}\end{array}$ & OFS-Asp & $\begin{array}{l}\text { Asp } \\
\mathrm{Na}\end{array}$ & $4 \mid Z M$ & & X-ray diffraction & 4.50 & 2013 & DOI: $10.1038 / n s m b .2548$ \\
\hline $\begin{array}{l}\mathrm{Glt}_{\mathrm{ph}} \\
\mathrm{K} 55 \mathrm{C} / \mathrm{A} 364 \mathrm{C}\end{array}$ & IFS-Apo-TI & $\mathrm{Tl}^{+}$ & $4 \mathrm{P} 1 \mathrm{~A}$ & & X-ray diffraction & 3.75 & 2014 & DOI: 10.7554/eLife.02283 \\
\hline $\begin{array}{l}\mathrm{Glt}_{\mathrm{ph}} \\
\mathrm{K} 55 \mathrm{C} / \mathrm{A} 364 \mathrm{C}\end{array}$ & IFS-Apo & & 4P19 & & X-ray diffraction & 3.25 & 2014 & DOI: 10.7554/eLife.02283 \\
\hline $\begin{array}{l}\text { Gltph } \\
\text { K55C/A364C }\end{array}$ & $\begin{array}{l}\text { IFS-Apo } \\
\text { alkali-free }\end{array}$ & & 4P3J & & X-ray diffraction & 3.50 & 2014 & DOI: 10.7554/eLife.02283 \\
\hline $\begin{array}{l}\mathrm{Glt}_{\mathrm{ph}} \\
\mathrm{K} 55 \mathrm{C} / \mathrm{A} 364 \mathrm{C}\end{array}$ & IFS-Bound-TI & $\mathrm{Tl}^{+}$ & $4 \mathrm{P} 6 \mathrm{H}$ & & X-ray diffraction & 4.08 & 2014 & DOI: 10.7554/eLife.02283 \\
\hline $\begin{array}{l}\text { Glt }_{\text {ph }} \\
\text { R397A }\end{array}$ & OFS-Na & $\mathrm{Na}$ & 4OYF & & X-ray diffraction & 3.41 & 2014 & DOI: 10.7554/eLife.02283 \\
\hline $\begin{array}{l}\text { Glt }_{\text {ph }} \\
\text { R397A }\end{array}$ & OFS-Apo & & 4OYE & & X-ray diffraction & 4 & 2014 & DOI: 10.7554/eLife.02283 \\
\hline $\begin{array}{l}\text { Glt } \\
\text { R276S/M395R }\end{array}$ & $\begin{array}{l}2 \text { IFS-Asp-unlocked } \\
1 \text { IFS-Asp }\end{array}$ & $\begin{array}{l}\text { Asp } \\
\mathrm{Na}\end{array}$ & $4 \times 2 S$ & & X-ray diffraction & 4.21 & 2015 & DOI: 10.1038/nature14158 \\
\hline $\mathrm{Glt}_{\mathrm{ph}}$ & OFS-Asp & $\begin{array}{l}\text { Asp } \\
\mathrm{Na}\end{array}$ & 6BAT & & X-ray diffraction & 3.40 & 2018 & DOI: 10.1038/s41467-017-02444-w \\
\hline $\begin{array}{l}\text { Glt }_{\text {ph }} \\
\text { R397C }\end{array}$ & IFS-Cysteine & $\begin{array}{l}\mathrm{Na} \\
\text { benzylcysteine }\end{array}$ & GBAV & & X-ray diffraction & 3.70 & 2018 & DOI: 10.1038/s41467-017-02444-w \\
\hline $\begin{array}{l}\text { Glt }_{\text {ph }} \\
\text { R397C }\end{array}$ & IFS-Serine & $\begin{array}{l}\mathrm{Na} \\
\text { Serine }\end{array}$ & $6 \mathrm{BMl}$ & & X-ray diffraction & 3.90 & 2018 & DOI: 10.1038/s41467-017-02444-w \\
\hline $\begin{array}{l}\text { Glt }_{\text {ph }} \\
\text { R397C }\end{array}$ & IFS-Cysteine & $\begin{array}{l}\mathrm{Na} \\
\text { cysteine }\end{array}$ & GBAU & & X-ray diffraction & 3.80 & 2018 & DOI: 10.1038/s41467-017-02444-w \\
\hline $\begin{array}{l}\text { Glt }_{\mathrm{ph}} \\
\text { R276S/M395R/ } \\
\text { K55C/A364C }\end{array}$ & IFS-Asp & $\begin{array}{l}\text { Asp } \\
\mathrm{Na}\end{array}$ & 6CTF & & X-ray diffraction & 4.05 & 2018 & DOI: 10.7554/eLife.37291 \\
\hline $\mathrm{Glt}_{\mathrm{ph}}$ & iOFS-Asp & Asp & 6UWL & EMD-20923 & Cryo-EM & 3.62 & 2020 & DOI: 10.1038/s41589-020-0561-6 \\
\hline $\mathrm{Glt}_{\mathrm{ph}}$ & OFS-Asp & Asp & 6UWF & EMD-20922 & Cryo-EM & 3.08 & 2020 & DOI: 10.1038/s41589-020-0561-6 \\
\hline $\mathrm{Glt}_{\mathrm{Ph}}$ & IFS-Asp & $\begin{array}{l}\mathrm{Na} \\
\mathrm{Asp}\end{array}$ & $6 \times 15$ & EMD-21989 & Cryo-EM & 3.05 & 2020 & DOI: 10.7554/eLife.58417 \\
\hline Glt $_{p h}$ & OFS-TBOA & & $6 \times 17$ & EMD-21991 & Cryo-EM & 3.66 & 2020 & DOI: 10.7554/eLife.58417 \\
\hline Glt $_{\mathrm{ph}}$ & IFS-TBOA & TBOA & $6 \times 16$ & EMD-21990 & Cryo-EM & 3.39 & 2020 & DOI: 10.7554/eLife.58417 \\
\hline $\mathrm{Glt}_{\mathrm{ph}}$ & IFS-TFBTBOA & TFBTBOA & $6 \times 14$ & EMD-21988 & Cryo-EM & 3.71 & 2020 & DOI: 10.7554/eLife.58417 \\
\hline Glt $_{\mathrm{ph}}$ & IFS-Na & & $6 \times 13$ & EMD-21987 & Cryo-EM & 3.66 & 2020 & DOI: 10.7554/eLife.58417 \\
\hline $\mathrm{Glt}_{\mathrm{ph}}$ & IFS-Apo-open & & $6 \times 12$ & EMD-21986 & Cryo-EM & 3.52 & 2020 & DOI: 10.7554/eLife.58417 \\
\hline $\mathrm{Glt}_{\mathrm{TK}}$ & OFS-Apo & & $4 \mathrm{KYO}$ & & X-ray diffraction & 3.00 & 2013 & DOI: 10.1038/nsmb.2663 \\
\hline $\mathrm{Glt}_{\mathrm{TK}}$ & OFS-Apo & & 5DWY & & X-ray diffraction & 2.70 & 2016 & DOI: 10.1038/ncomms13420 \\
\hline $\mathrm{Glt}_{\mathrm{TK}}$ & OFS-NaAsp & $\begin{array}{l}\mathrm{Na} \\
\mathrm{Asp}\end{array}$ & 5E9S & & X-ray diffraction & 2.80 & 2016 & DOI: 10.1038/ncomms13420 \\
\hline $\mathrm{Glt}_{\mathrm{TK}}$ & OFS-Asp & $\begin{array}{l}\mathrm{Na} \\
\mathrm{D}-\mathrm{Asp}\end{array}$ & 6R7R & & X-ray diffraction & 2.80 & 2019 & DOI: 10.7554/eLife.45286 \\
\hline
\end{tabular}


TABLE 1 | Continued

\begin{tabular}{|c|c|c|c|c|c|c|c|c|}
\hline Protein & State & Ligand & $\begin{array}{l}\text { PDB } \\
\text { code }\end{array}$ & EMDB id & $\begin{array}{l}\text { Structure } \\
\text { determination } \\
\text { method }\end{array}$ & $\begin{array}{l}\text { Resolu- } \\
\text { tion }(\AA)\end{array}$ & $\begin{array}{l}\text { Year of } \\
\text { release }\end{array}$ & Reference \\
\hline $\mathrm{Glt}_{\mathrm{TK}}$ & $\begin{array}{l}2 \text { OFS-TBOA } \\
1 \text { IFS-Na }\end{array}$ & TBOA & 6XWN & EMD-10632 & Cryo-EM & 3.47 & 2020 & DOI: $10.1038 / s 41467-020-14834-8$ \\
\hline $\mathrm{Glt}_{T \mathrm{~K}}$ & $\begin{array}{l}2 \text { iOFS-Asp } \\
1 \text { IFS-Na }\end{array}$ & Asp & 6XWP & EMD-10634 & Cryo-EM & 3.38 & 2020 & DOI: $10.1038 / s 41467-020-14834-8$ \\
\hline $\mathrm{Glt}_{\mathrm{TK}}$ & $\begin{array}{l}1 \text { iOFS-Asp } \\
2 \text { IFS-Na }\end{array}$ & Asp & 6XWO & EMD-10633 & Cryo-EM & 3.39 & 2020 & DOI: $10.1038 / s 41467-020-14834-8$ \\
\hline $\mathrm{Glt}_{T \mathrm{~K}}$ & $\begin{array}{l}1 \text { iOFS-Apo } \\
2 \text { IFS-Na }\end{array}$ & & 6XWR & EMD-10636 & Cryo-EM & 3.22 & 2020 & DOI: $10.1038 / s 41467-020-14834-8$ \\
\hline $\mathrm{Glt}_{\mathrm{TK}}$ & iOFS-Asp & Asp & $6 X W Q$ & EMD-10635 & Cryo-EM & 3.41 & 2020 & DOI: $10.1038 / s 41467-020-14834-8$ \\
\hline $\begin{array}{l}\text { EAAT1 } \\
\text { Cryst mutant }\end{array}$ & OFS-Asp & $\begin{array}{l}\mathrm{Na} \\
\text { Asp } \\
\text { UCPH101 }\end{array}$ & $5 \mathrm{LLM}$ & & X-ray diffraction & 3.25 & 2017 & DOI: 10.1038/nature22064 \\
\hline $\begin{array}{l}\text { EAAT1 } \\
\text { Cryst mutant }\end{array}$ & OFS-TFBTBOA & $\begin{array}{l}\text { TFB-TBOA } \\
\text { UCPH101 }\end{array}$ & $5 \mathrm{MJU}$ & & X-ray diffraction & 3.71 & 2017 & DOI: 10.1038/nature22064 \\
\hline $\begin{array}{l}\text { EAAT1 } \\
\text { Cryst mutant }\end{array}$ & OFS-Asp & $\begin{array}{l}\mathrm{Na} \\
\mathrm{Asp}\end{array}$ & $5 L L U$ & & X-ray diffraction & 3.32 & 2017 & DOI: 10.1038/nature22064 \\
\hline
\end{tabular}

a.
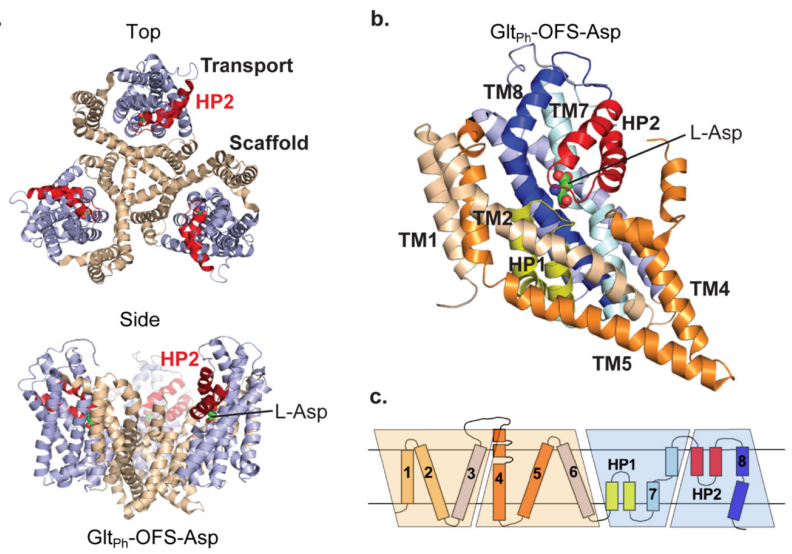

FIGURE 1 I Structure of glutamate transporter homolog Glt $_{\mathrm{Ph}}$ (A) View of Glt $_{\text {ph }}$-OFS-Asp (PDB 2NWL) trimer from the extracellular side of the membrane (top) or in the plane of the membrane (side). The trimer is shown in cartoon representation. The scaffold domain is colored wheat, the transport domain blue and HP2 red. (B) One monomer of Glt ${ }_{p h}$ shown in cartoon representation colored based on the topology color scheme in (C) Substrate Asp is shown in sphere representation. (C) Schematic representation of $\mathrm{Glt}_{\mathrm{ph}}$ transmembrane topology. The two inverted repeats segments are colored as transparent yellow and blue, respectively.

excluded from exposure to the extracellular solution merely by HP2. In contrast, the bound substrate and the cytoplasm are separated by over $15 \AA$ of a compact transport domain core. Therefore this structure is determined as a closed outwardfacing state (OFS) of the transporter (Glt $\mathrm{Ph}_{\mathrm{Ph}}$-OFS-Asp) (Figure 1A, B).

A remarkable feature of the structure of $\mathrm{Glt}_{\mathrm{Ph}}$ is it contains two sets of the so-called inverted structural repeats (Figure 1C). Inverted repeats are structural elements that are approximately related by a twofold symmetry and are positioned in the membrane in an antiparallel orientation. The first repeat includes the scaffold TMs and two TMs from the transport domain: TMs 1, 2 , and 3 can be superimposed onto TMs 4,5 , and 6 by rotation around an axis parallel to the membrane. The second repeat is within the transport domain: HP1 and TM7 can be superimposed onto HP2 and TM8 by around $145^{\circ}$ rotation. By switch of the relative orientation of the repeat in the transport domain relative to the first, the conformational transition between the outward to inward-facing states was approximated (Crisman et al., 2009) and revealed by a crystal structure of the inward-facing state (IFS) of Glt $t_{\text {Ph }}$ described below (Reyes et al., 2009).

\section{ELEVATOR MECHANISM OF GLUTAMATE TRANSPORT}

Transporter protein translocates substrate by alternating its access to either the extracellular milieu or the cytoplasm. Several studies on EAATs have investigated the protein conformational changes during glutamate transport (Focke et al., 2011, Koch and Larsson, 2005). Notably, in an EAAT1 study, two residues that are far away apart (more than $25 \AA$ ) based on the outward-facing state of the Glt $t_{\mathrm{ph}}$ homolog were shown to form a spontaneous disulfide bond (Ryan et al., 2004). This surprising result suggests these residues could get close, reflecting unknown functional state of the transporter. By engineering the corresponding residues K55/A364 in Glt $_{\mathrm{Ph}}$ into cysteine and crosslinking them with $\mathrm{Hg}^{2+}$, a crystal structure was solved with the transporter being trapped in a new state (Glt ph -IFS-Asp) (Reyes et al., 2009). In this novel structure, the transport domain moves $15 \AA$ across the bilayer, while the scaffold domain remains largely in place (Figure 2). The substrate and ion-binding sites, which are positioned entirely within the transport domain, are preserved. Because the bound Asp is still completely occluded from the solvent but is near the intracellular solution, the structure represents an inward-facing state (IFS) of the transporter. In Glt $\mathrm{Ph}_{\mathrm{Ph}}$-OFS-Asp, HP1 and the cytoplasmic 


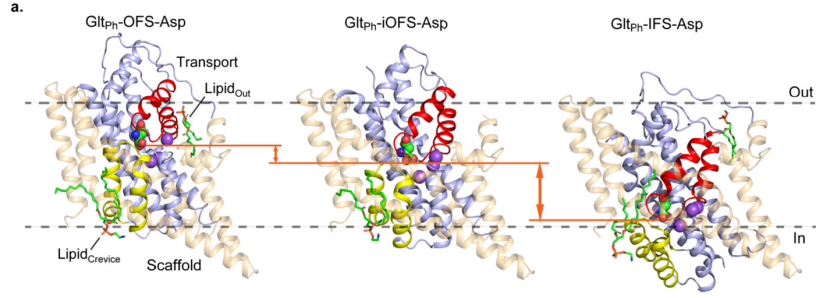

FIGURE 2 I Substrate and $\mathrm{Na}^{+}$ions are carried across the lipid bilayer from OFS to IFS by an elevator-like mechanism with an iOFS in between. Glt $_{\mathrm{Ph}}$-OFS-Asp (PDB 6UWF), Glt $\mathrm{Ph}_{\mathrm{ph}}$-iOFS-Asp (PDB 6UWL) and Glt $\mathrm{Ph}_{\mathrm{Ph}}$-IFSAsp (PDB 6X15) are aligned on TM4c and the first half of TM5 of the scaffold domain. Single protomers are shown in cartoon representation. The scaffold domain is colored wheat, the transport domain blue, HP1 yellow and HP2 red. Asp and $\mathrm{Na}^{+}$are shown in sphere representation, lipids in stick representation. The orange line indicates the position of the bound substrate Asp.

portion of TM7 are packed against the scaffold. In Glt $_{\mathrm{Ph}}$-IFSAsp, the interaction with the scaffold is replaced by HP2 and the extracellular segment of TM8 instead (Figure 2). The transporter works like an "elevator": substrate translocation across the lipid bilayer is achieved by the rigid movement of a transport domain relative to a scaffold domain. Later structural studies show that several transporters that are not closely related to the SLC1A family also function with similar mechanisms (Garaeva and Slotboom, 2020). Following functional studies on $\mathrm{Glt}_{\mathrm{Ph}}$ and $\mathrm{Glt}_{\mathrm{Tk}}$ using electron paramagnetic resonance (EPR), single-molecule FRET (smFRET) and high-speed atomic force microscopy (HSAFM) measured the "elevator-like" large-scale transport domain movement and state distributions between the OFS and IFS (Akyuz et al., 2013; Erkens et al., 2013; Georgieva et al., 2013; Hänelt et al., 2013; Ruan et al., 2017). For wild type (WT) Glt in lipidic environment, the transition from OFS to IFS appears to be the rate-limiting step in the presence of Asp and $\mathrm{Na}^{+}$. The competitive transport blocker DL-threo- $\beta$-benzyloxyaspartate (TBOA) stabilized OFS and reduced OFS/IFS dynamics (Akyuz et al., 2013; Ruan et al., 2017)

In 2012, another crosslinked structure of $\mathrm{Glt}_{\mathrm{Ph}}-\mathrm{V} 198 \mathrm{C} / \mathrm{A} 380 \mathrm{C}$ pictured an asymmetric trimer with two protomers in the IFS and the third in an intermediate outward-facing state (iOFS) (Figure 2) (Verdon and Boudker, 2012). It was speculated that the novel conformation observed has been captured because the iOFS is a relatively low energy state. Still, the unique crystal packing contacts for this mutant might have stabilized this conformation. Until recently, a Cryo-EM study on WT GIt reconstituted in lipidic nanodisc shows that after processing the data with symmetry expansion (C3) and 3D classification on one single protomer, besides the majority of the protomersbeing in the OFS state, around $10 \%$ of the molecules were indeed in the iOFS conformation (Huang et al., 2020). A similar result from $\mathrm{Glt}_{\mathrm{TK}}$ solved in nanodisc revealed the existence of iOFS as well, except that in $\mathrm{Glt}_{\mathrm{Tk}}$ in the lipidic environment, iOFS seems to be a preferred state over OFS (Arkhipova et al., 2020). Lately, a high-speed atomic force microscopy line-scanning (HS-AFM-LS) study shows in Glt $_{\mathrm{ph}}$ an intermediate state exits with measurable a.

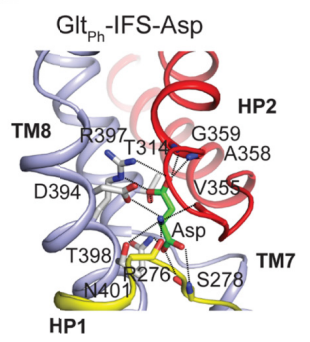

c.

b.

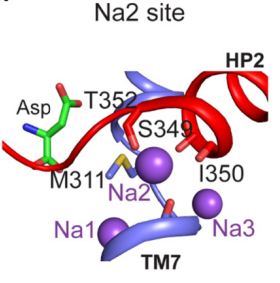

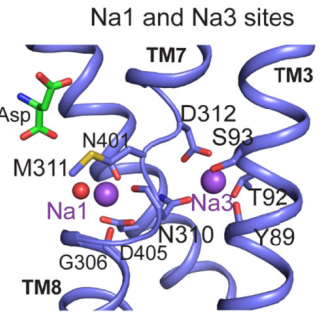

d.

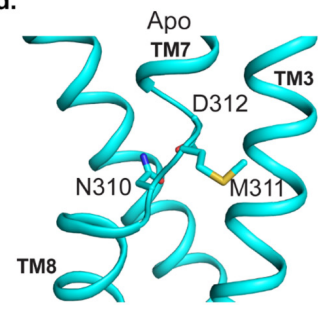

FIGURE 3 I Substrate and $\mathrm{Na}^{+}$binding sites. (A) Close-up view of the substrate-binding pocket of Glt $\mathrm{ph}_{\mathrm{p}}$-IFS-Asp (PDB 6X15) with bound Asp shown in stick representation and colored by atom type. Dotted lines correspond to potential hydrogen bonds. Figure is modified from (Wang and Boudker, 2020) with permission. (B) and (C). Structures of the Na1, Na2 and Na3 sites in $\mathrm{Glt}_{\mathrm{Ph}}{ }^{-}$ IFS-Asp (PDB 6X15). Coordinating moieties within $3 \AA$ of the ions are shown as sticks. $\mathrm{Na}^{+}$ions and a resolved water molecule are shown as purple and red spheres, respectively. (D) NMDGT motif on TM7 turns away in Glt $\mathrm{Ph}_{\mathrm{Ph}}-\mathrm{IFS}-\mathrm{Apo}$ structure (PDB 4P19).

kinetics between OFS and IFS (Matin et al., 2020). Though it is unknown if the intermediate state observed in HS-AFM-LS study is the iOFS solved in the structural study. Not alone, a recent ${ }^{19} \mathrm{~F}-\mathrm{NMR}$ spectroscopy study also presents two outward-facing states in Glt $_{\mathrm{Ph}}$ measured in a detergent environment (Huang et al., 2020).

After releasing Asp and $\mathrm{Na}^{+}$into the cytoplasm, the inwardfacing apo transporter could reorient back to OFS, to accomplish the full transport cycle. The crystal or Cryo-EM structures of Glt and $\mathrm{Glt}_{\mathrm{TK}}$ in apo conditions were solved in the OFS, iOFS, and IFS. They showed similar positions of the transport domains as the bound states. There are local structural rearrangements in the apo conformations associated with the release of the bound Asp and $\mathrm{Na}^{+}$ions discussed in the following text (Verdon et al., 2014; Jensen et al., 2013).

\section{SUBSTRATE AND NA+ BINDING SITES}

In Asp bound structures, Asp is completely buried within a polar chamber formed by the tips of HP1 and HP2, the unwound $\beta$-bridge region of TM7 (NMDGT motif) and polar residues of amphipathic TM8 (Figure 3A) (Boudker et al., 2007). The amino group of Asp is coordinated by R276 (HP1), V355 (HP2), D394 (TM8) and T398 (TM8). The carboxylate group of Asp interacts with S278 (HP1) and N401 (TM8). The $\beta$-carboxylate interacts with T314 (TM7), G359 (HP2), A358 (HP2) and R397 (TM8) (PDB 6X15) (Figure 3A). These regions were previously implicated in substrate binding and translocation (Slotboom et al., 2001). Residues involved in aspartate coordination are 
mostly conserved. Notably, In dicarboxylate transporters, D394 is substituted with a serine residue (Engelke et al., 1989; Yurgel and Kahn, 2005). Mutation of R397 to cysteine or threonine in mammalian transporters abolish acidic amino-acid transport (Conradt and Stoffel, 1995; Seal et al., 2000), whereas neutral amino-acid exchange is introduced (Bendahan et al., 2000). Mutation of the equivalent residue T459 in the neutral amino acid transporter ASCT1 to arginine alters the substrate specificity to prefer acidic amino acids. In addition, substituting the equivalent residue in Glt $t_{\mathrm{ph}}$ to its ASCT2 counterpart (R397C) switches the substrate selectivity from acidic to neutral amino acids (Scopelliti et al., 2018).

The binding sites of two of the three coupled $\mathrm{Na}^{+}$ions were revealed by a crystal structure of $\mathrm{Glt}_{\mathrm{ph}}$ solved in the presence of Asp and $\mathrm{TI}^{+}$ions in 2007 (Figure 3B, C) (Boudker et al., 2007). They were named $\mathrm{Na} 1$ and $\mathrm{Na} 2$, respectively. $\mathrm{Na} 1$ site is buried deep under the bound $\mathrm{Asp}$ and the $\mathrm{Na}^{+}$ion is mainly coordinated by the side chain of D405 in TM8. Other residues that participate in coordinating Na1 are main chain oxygens of G306, N310 on TM7, N401 on TM8, and one water molecule. Na2 site is formed by main chain oxygens of S349, I350 and T352 on the c-terminal end of the HP2a helix and T308 on the TM7. The location of the third $\mathrm{Na}^{+}$ion, first predicted based on computational and mutagenesis studies (Zarbiv et al., 1998; Tao et al., 2010; Bastug et al., 2012; Silverstein et al., 2013), has been observed in the structure of $\mathrm{Glt}_{\mathrm{Tk}}$ in 2016 (Guskov et al., 2016). $\mathrm{Na}^{+}$at the $\mathrm{Na} 3$ site in Glt $_{\mathrm{Ph}}$ is coordinated by side chains of N310 and D312 on the extended NMDGT motif of TM7, together with Y89, T92 and S93 on TM3. Compared with Asp/ $\mathrm{Na}^{+}$bound conformations, apo states of $\mathrm{Glt}_{\mathrm{Tk}}$ and $\mathrm{Glt} \mathrm{t}_{\mathrm{Ph}}$ show distortion of all three $\mathrm{Na}$ sites. Notably, the NMDGT motif is restructured that the M311 side chain, instead of pointing toward the Asp and $\mathrm{Na} 2$ binding sites, now turns away and points toward TM3, into the $\mathrm{Na} 3$ site. There is also a noticeable bend of TM3 to accommodate the M311 rearrangement. It looks like the NMDGT motif is vital in coordinating $\mathrm{Na}^{+}$ions and couple the conformation change induced by $\mathrm{Na}^{+}$binding to accommodate substrate binding.

\section{GATING MECHANISM OF GLUTAMATE TRANSPORTERS}

Both the OFS and IFS Asp-bound structures discussed above represent occluded states of glutamate transporters where the substrate is prevented from accessing either the extracellular or the cytoplasm solution. A clue to the identity of the extracellular gate came from solving the crystal structure of $\mathrm{Glt}_{\mathrm{Ph}}$ in complex with the non-transportable blocker TBOA in 2007 (Glt $\mathrm{t}_{\mathrm{Ph}}$-OFSTBOA) (Boudker et al., 2007). The overall structure of the transporter is similar in the Glt $\mathrm{ph}_{\mathrm{ph}}$-OFS-Asp and Glt $\mathrm{ph}_{\mathrm{p}}$-OFS-TBOA complexes with the exception of HP2 region (Figure 4A). In the a.

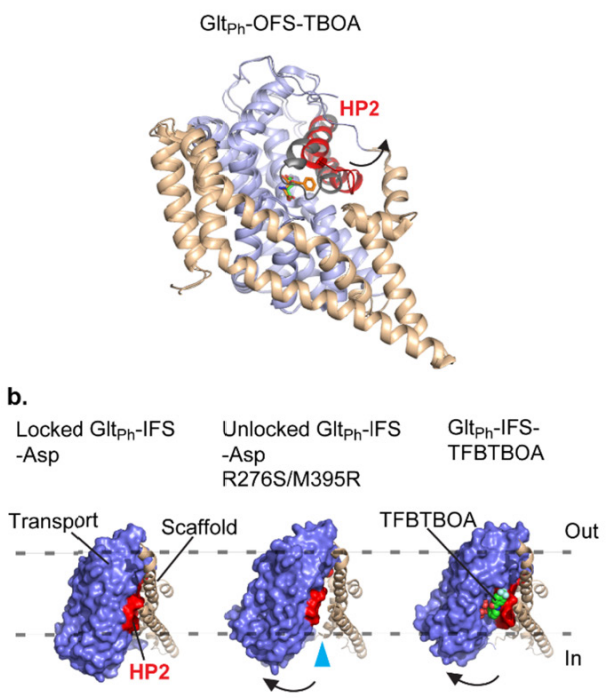

c.
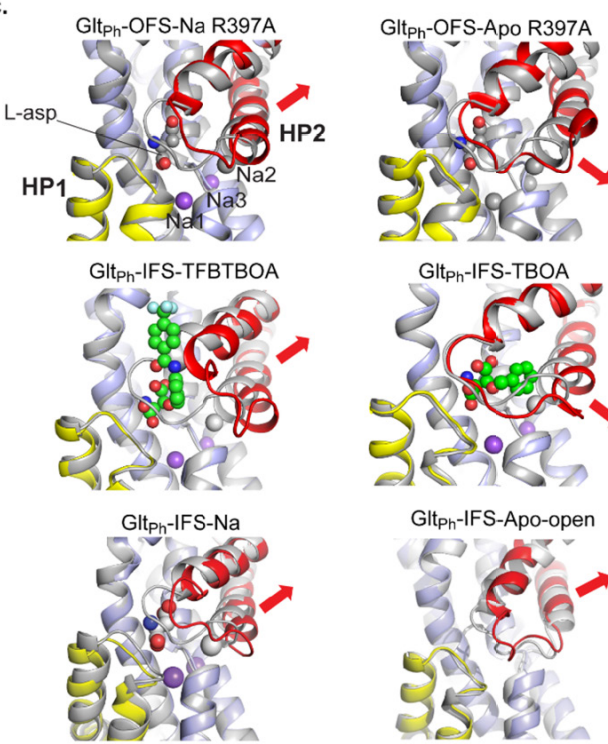

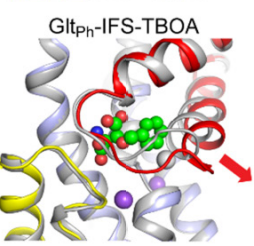

GIt $_{\text {Ph }}$ IFS-Apo-open

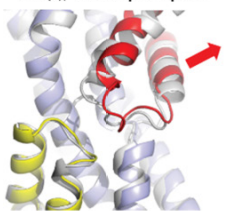

FIGURE 4 I Gating in OFS requires only HP2 opening, while gating in IFS requires HP2 opening coupled to a large swing-out movement of the rest of the transport domain. (A) Superimposition of Glt $\mathrm{p}_{\mathrm{p}}$-OFS-TBOA (PDB 2NWW) and Glt $\mathrm{p}_{\mathrm{p}}$-OFS-Asp (PDB 6UWF). The scaffold domain is colored wheat, the transport domain blue. HP2 in Glt $t_{p h}-$ OFS-TBOA is colored red and HP2 in Glt $p_{p h}$-OFS-Asp is colored grey. The black arrow indicates the opening of HP2. TBOA and Asp are shown in stick representation. (B) Glt $t_{p h}$-IFS-Asp (PDB 6X15), the unlocked protomer of Glt $t_{p h}$-IFS-Asp R276S/M395R (PDB 4X2S), and Glt 6X14) are aligned on the scaffold domain. The transport domain is shown in surface representation and the scaffold domain cartoon representation. TFBTBOA is shown as spheres. Black arrows indicate the swing-out movement of the transport domain. Cyan arrow marks the enlarged crevice between the transport and scaffold domain. (C) Glt $\mathrm{ph}_{\mathrm{h}}$-OFS-Na R397A (colored) (PDB 4OYF) and Glt $\mathrm{ph}_{\mathrm{h}}$-OFS-Apo R397A (colored) (PDB 4OYE) are superimposed with Glt $\mathrm{ph}_{\mathrm{ph}}$-OFS-Asp (grey) (PDB 6UWF) on TM7 of the transport domain. Glt ${ }_{\mathrm{ph}}-\mathrm{IFS}-\mathrm{TFBTBOA}$ (colored) (PDB 6X14), Glt $\mathrm{t}_{\mathrm{ph}}$-IFS-TBOA (colored) (PDB 6X16), and Glt $\mathrm{ph}_{\mathrm{ph}}-\mathrm{IFS}-\mathrm{Na}$ (colored) (PDB 6X13)

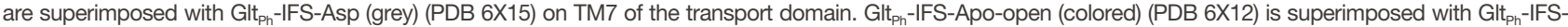
Apo (grey) (PDB 4P19) on TM7 of the transport domain. Asp, TFBTBOA, TBOA and $\mathrm{Na}^{+}$are shown in sphere representation. Red arrows indicate the direction of movement of HP2. Yellow arrow indicates the movement of HP1. The Glt $\mathrm{ph}_{\mathrm{H}} \mathrm{IFS}-\mathrm{Na}$ and GltPh-IFS-Apo-open figures are modified from (Wang and Boudker, 2020) with permission. 
a.

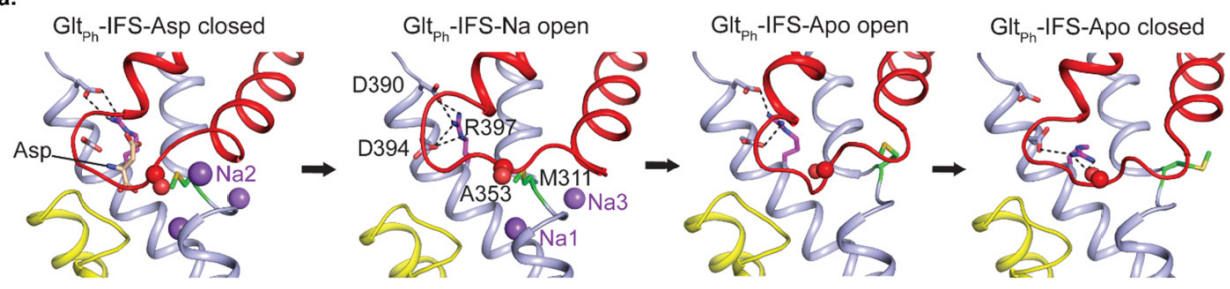

FIGURE 5 I Substrate and $\mathrm{Na}^{+}$coupled gating. (A) Gating steps in the inward-facing state of Glt $\mathrm{ph}_{\mathrm{p}}$. Local structural changes from Asp-closed (PDB 6X15) to Na+open (PDB 6X13) state, then to Apo-open (PDB 6X12), and finally to Apo-closed (PDB 4P19) state. The structures are in cartoon representation. TM7 and TM8 are colored light blue, HP1 colored yellow and HP2 colored red. Substrate Asp and key residues are in stick representation and colored by atom type. A353 is shown in sphere representation. Dotted lines correspond to potential hydrogen bonds.

a.

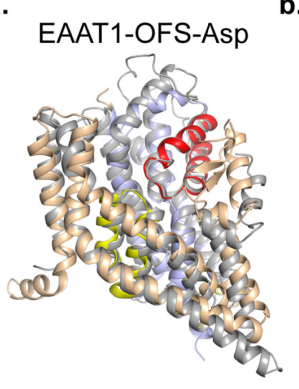

b. EAAT1-OFS-Asp in UCPH101

FIGURE 6 I EAAT1 structure shows high similarity with GItPh and reveals the unique binding of an inhibitor UCPH101. (A) Superimposition of EAAT1OFS-Asp (PDB 5LLU) with Glt ph $_{\mathrm{h}}$-OFS-Asp (PDB 6UWF). The scaffold domain of EAAT1 is colored wheat, the transport domain blue. HP1 yellow and HP2 red. GItPh-OFS-Asp is colored grey. (B) EAAT1-OFS-Asp in the presence of UCPH101 (PDB 5LLM). Asp, Na2 and UCPH are shown in sphere representation.

TBOA-bound structure, HP2 adopts an 'open' conformation, moving as much as $10 \AA$ from its position in the aspartate-bound complex, exposing the substrate-binding site to the extracellular solution (Figure 4A). The aspartate moiety of TBOA binds in the substrate-binding site, in agreement with the observation that TBOA is a competitive inhibitor and the bulky benzyl group of TBOA lodges against the tip of HP2, propping it in an open conformation. Therefore, HP2 functions as the extracellular gate of the glutamate transporter. It looks like Asp binding needs to be preceded by an opening of HP2, exposing the substrate-binding site. In TBOA-bound structure, both the $\mathrm{Na} 1$ and $\mathrm{Na} 3$ sites seem to be in $\mathrm{Na}^{+}$-coordinating positions, while the $\mathrm{Na} 2$ site is distorted due to the opening of HP2, in line with studies showing TBOA binding is coupled to the binding of two $\mathrm{Na}^{+}$ions (Reyes et al., 2013; Oh and Boudker, 2018). Interestingly, HP2 was also in the same open conformation in an R397C Glt $_{\mathrm{Ph}}$ mutant bound to glutamine or benzyl-cysteine (Scopelliti et al., 2018). In these structures, there are no direct interaction between the ligands and HP2 but steric clashes disallow closure. Therefore, it appears that HP2 intrinsically favors this open conformation. It looks like OFS with open HP2 is unable to isomerize to IFS, as the HP2 opening would clash with the scaffold domain during translocation to IFS. In 2014, a Glt $\mathrm{ph}_{\mathrm{ph}}$ R397A mutant was crystallized in $\mathrm{Na}^{+}$only condition (Verdon et al., 2014). In this

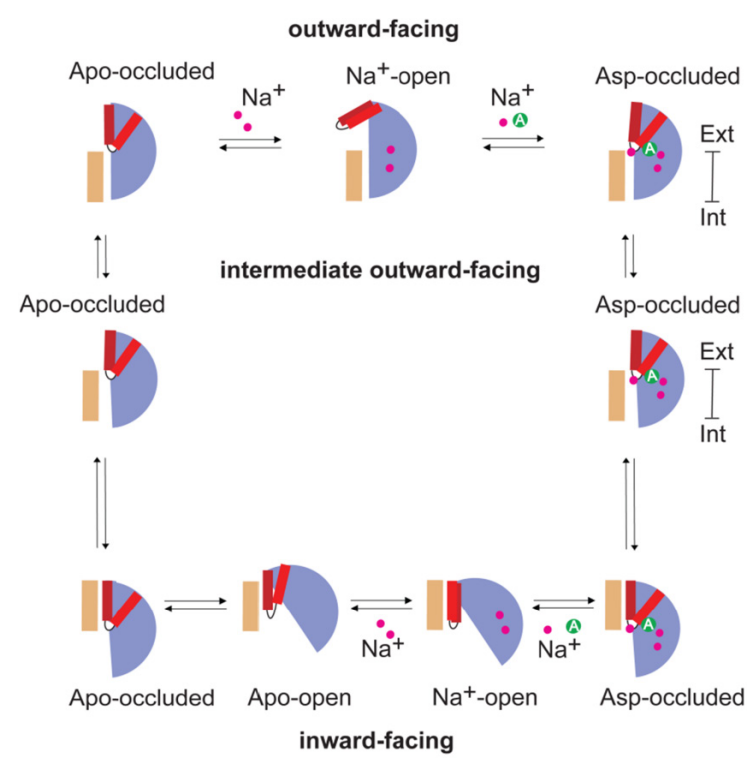

FIGURE 7 I A schematic representation of the transport mechanism of glutamate transporter based on structural studies on $\mathrm{Glt}_{\mathrm{Ph}}$ and $\mathrm{Glt}_{\mathrm{Tk}}$. One monomer of the transporter is shown. The scaffold domain is colored wheat, the transport domain blue and HP2 red. The transported Asp is shown as a green " $A$ ".

Glt $t_{\text {ph }}$-OFS-Na structure, HP2 acquire a less open conformation compared with $\mathrm{Glt}_{\mathrm{Ph}}$-OFS-TBOA, probably due to the mutation disturbing HP2 coupling with Na binding (Figure 4C). Also, OFSApo crystal structures were reported in $\mathrm{Glt}_{\mathrm{TK}}$ and $\mathrm{Glt} \mathrm{t}_{\mathrm{Ph}}$ (Jensen et al., 2013; Verdon et al., 2014). In both apo structures, HP2 assumes closed conformation, with the c-terminal of HP2a helix unwounded and therefore Na2 site distorted (Figure 4C). Altogether, these studies suggest the sequential events upon $\mathrm{Na}^{+}$and Asp binding in OFS: Starting from HP2 closed apo state, $\mathrm{Na}^{+}$binding at $\mathrm{Na} 1$ and $\mathrm{Na} 3$ open HP2, the following binding of Asp closes HP2, HP2 closure is further secured by $\mathrm{Na}^{+}$binding at $\mathrm{Na} 2$, and the later step of the transport cycle is initiated (Figure 7).

Gating in the IFS was initially believed to involve the opening of the structurally symmetric HP1 (Boudker et al., 2007). However, HP1 lacks the abundance of glycine residues which confers the flexibility of HP2. A clue of the IFS gating mechanism comes 
from a gain of function Glt $\mathrm{Ph}_{\mathrm{ph}}$ mutant R276S/M395R, in which two protomers are found in an "unlocked" conformation (Akyuz et al., 2015). The transport domain leans away from the scaffold, leaving a dramatically more open space between the two, allowing lipid and water access (Figure 4B). Most importantly, in this "unlocked" conformation, there is room for HP2 to open. Only recently, Cryo-EM studies on ASCT2, a neutral amino acid transporter of the SLC1A family, and also on $\mathrm{Glt}_{\mathrm{Tk}}$ and $\mathrm{Glt}_{\mathrm{Ph}}$ have shown that gating in the IFS indeed involves the opening of HP2 (Garaeva et al., 2019, Arkhipova et al., 2020, Wang and Boudker, 2020). The inward-gating mechanism of glutamate transporters is most extensively explored in Cryo-EM studies of IFS GIt in nanodisc and therefore used as the examples here. In $\mathrm{Glt}_{\mathrm{Ph}}{ }^{-}$ IFS bound with another competitive inhibitor (2S,3S)-3-[3-[4(trifluoromethyl)benzoylamino]benzyloxy]aspartate (TFBTBOA), the bound TFBTBOA shares the same binding pocket as Asp. However, its hydrophobic side chain lodges against the second half of the HP2 helix, leaving HP2 wildly open (Figure 4B, C). Strikingly, to accommodate HP2 opening, the transport domain swung away further more than observed in Glt $_{\mathrm{Ph}}$ R276S/M395R. When aligned on the transport domain alone, Glt $\mathrm{t}_{\mathrm{ph}}$-IFS-TFBTBOA is nearly identical with Glt $t_{\mathrm{ph}}-\mathrm{OFS}-\mathrm{TBOA}$. Therefore, it seems that HP2 also serves as the gate in the IFS; its opening is coupled to the leaning away of the transport domain from the scaffold. Indeed, both the $\mathrm{Na}^{+}$-only GIt $\mathrm{Ph}_{\mathrm{Ph}}$-IFS-Na and GItPh-IFS-Apo-open structures show various degrees of swing-out movement of the transport domain coupled to the opening and local structural rearrangements of HP2 (Figure 4C) (Wang and Boudker, 2020). The conformation of HP2 region itself displays a high degree of plasticity depending on the presence of Asp, inhibitors, $\mathrm{Na}^{+}$only or apo.

Similar to OFS, in IFS HP2 could switch between closed and open conformations. Once HP2 opens, $\mathrm{Na}^{+}$binding at $\mathrm{Na} 1$ and $\mathrm{Na} 3$ site stabilizes the HP2 open conformation, and the following binding of Asp and $\mathrm{Na}^{+}$at $\mathrm{Na} 2$ close the HP2 (Figures 5 and 7). It is unknown whether the transport domain, together with HP2, swings out as a bundle first, then HP2 opens, or HP2 always stays packed against the scaffold and the rest of the transport domain moves away, exposing the substratebinding site. Another interesting point is in the Glt $\mathrm{tph}_{\mathrm{ph}}$-IFS in the presence of $\mathrm{Na}^{+}$and TBOA (GIt $\mathrm{ph}_{\mathrm{Ph}}$-IFS-TBOA), HP2 is in a closed conformation, in contrast to the wide HP2 opening seen in the presence of TFBTBOA (Wang and Boudker, 2020). The benzyl group of TBOA resides in between the two arms of HP2, packed against M311 and M362 side chains. The N-terminal arm of HP2 shifts away, distorting the Na2 binding site and making space to accommodate the TBOA benzyl ring (Figure 4C). This new conformation might reflect a transient state where $\mathrm{Na}^{+}$ has left $\mathrm{Na} 2$ site while the substrate and the other two $\mathrm{Na}^{+}$are still bound. To be noted, this new mode of inhibitor binding in glutamate transporter, though only seen in IFS to date, might also exist in the OFS.

The transition from OFS to IFS requires breaking the interface between HP1/TM7 of the transport domain and the scaffold to destabilize the OFS, and forming the new interface between HP2/TM8a and the scaffold to stabilize the IFS. Different ligand and ion conditions result in the local structure rearrangement of HP2, altering its interactions with the scaffold domain in the IFS. Comparison of the domain interface area in the IFS of Glt $t_{\mathrm{ph}}$ solved in the presence of apo, $\mathrm{Na}^{+}$-only, Asp, or inhibitors show that the interface area of translocation-deficient states like $\mathrm{Glt}_{\mathrm{Ph}}$-IFS-Na and GIt $\mathrm{Ph}_{\mathrm{Ph}}$-IFS-TFBTBOA is much larger than translocation-competent states like $\mathrm{Glt}_{\mathrm{Ph}}$-IFS-Asp and $\mathrm{G}_{\mathrm{Ph}}$ IFS-Apo (Wang and Boudker, 2020). The altered geometry of the interface and larger interaction area may explain why translocation is inhibited by blockers or in $\mathrm{Na}^{+}$ions only, even though it is not possible to translate interaction areas into energies.

\section{HP2 GATING COUPLED TO ION AND SUBSTRATE BINDING}

Substrate and $\mathrm{Na}^{+}$binding, as discussed above, is coupled to the sequential structural changes of HP2 and the rest of the transport domain. It looks like M311 and R397 in the transport domain serve as the two "master residues" that move significantly during gating and couple solute binding and release to large-scale conformational changes. Here we take the structural events, which might underlie ion and substrate release in the IFS as an example (gating in OFS follows similar steps when exampled on the transport domain itself). Starting with $\mathrm{Glt}_{\mathrm{ph}}$-IFS-Asp and going to $\mathrm{Glt}_{\mathrm{Ph}}$-IFS-Na, Glt $\mathrm{Ph}_{\mathrm{Ph}}$-IFS-Apo-open, and Glt $\mathrm{Ph}_{\mathrm{Ph}}$-IFS-Apo-closed, in $\mathrm{Glt}_{\mathrm{Ph}}$-IFS-Asp, the R397 side chain extends upward toward the extracellular side. D390 coordinates its guanidinium group. In this position, R397 makes space for Asp and coordinates its sidechain carboxylate, while D394 coordinates its amino group (Figure 5). M311 protrudes into the binding site and coordinates $\mathrm{Na} 2$ (Figure 3B). Extensive interaction of HP2 with the bound Asp and Na2 favor the closed conformation (Figure 3A, B). HP2 opening allows Asp and $\mathrm{Na} 2$ release (Glt $\mathrm{t}_{\mathrm{Ph}}$-IFS-Na). R397 is now clamped between D390 and D394, while M311 remains in place (Figure 5). The consequent release of $\mathrm{Na} 1$ and $\mathrm{Na} 3$ leads to a Glt $_{\mathrm{Ph}}$-IFS-Apo-open conformation, where the NMDGT motif restructured and $M 311$ rotates outward, now packing against the open HP2 (Figure 5). The guanidinium group of R397 remains upward between D390 and D394. To achieve the closed apo state, M311 swings further out into the lipid bilayer, allowing HP2 to close. R397 descends into the binding pocket, coordinated only by D394, and is poised to make interactions with carbonyl oxygens of A353 on the tip of closed HP2. R397 entering the Asp-binding site and HP2 closure is prevented by steric hindrance of M311 and more positive local electrostatics in $\mathrm{Glt}_{\mathrm{Ph}}{ }^{-}$ IFS-Na, to prevent $\mathrm{Na}^{+}$leaks.

\section{STRUCTURE OF HUMAN GLUTAMATE TRANSPORTER EAAT1}

In 2017, the mammalian glutamate transporter's first structure was solved by crystalizing a functional EAAT1 thermostabilized 
mutant, which shares an overall $75 \%$ sequence identity with WT (Canul-Tec et al., 2017). The crystal structure resembles an EAAT1 in OFS, bound with Asp and $\mathrm{Na}^{+}$. Overall, the EAAT1OFS-Asp shows a high structural similarity with $\mathrm{Glt}_{\mathrm{Ph}}$-OFSAsp (RMSD: $1.5 \AA$ ) (Figure 6A). Substrate and $\mathrm{Na}^{+}$binding sites are also conserved between the two. An additional EAAT1 TFBTBOA-bound conformation shows that HP2 assumes an open conformation as in Glt $\mathrm{ph}_{\mathrm{ph}}$-OFS-TBOA, suggesting HP2 also serves as the extracellular gate in EAAT1 (Canul-Tec et al., 2017). Also, an allosteric EAAT1-selective inhibitor 2-amino-4-(4methoxyphenyl)-7-(naphthalen-1-yl)-5-oxo-5,6,7,8-tetrahydro$4 \mathrm{H}$-chromene-3-carbonitrile (UCPH101) is co-crystalized with EAAT1 in the presence of Asp or TFBTBOA (Canul-Tec et al., 2017). In both UCPH101 bound structures, the protein resumes an OFS, and UCPH10 densities were found in a hydrophobic pocket between TM3, TM7 and TM4c at the transport/scaffold domain interface (Figure 6B). The position of UCPH101 in Asp or TFBTBOA- bound structures are the same, far away from the substrate and $\mathrm{Na}^{+}$sites, in agreement with $\mathrm{UCPH}$ being an allosteric inhibitor. The compound extends the interface by around $500 \AA^{2}$, and is shown to stabilize the OFS by hydrogendeuterium exchange mass spectrometry (HDX-MS) (Canul-Tec et al., 2017).

\section{THE INTERPLAY BETWEEN GLUTAMATE TRANSPORTER AND THE LIPID ENVIRONMENT}

In glutamate transporter structures solved in either detergent or lipid nanodiscs, a few densities represent detergent or lipid molecules are found in similar positions. These positions include the crevices between the $\mathrm{N}$-terminal helix and the rest of the scaffold ( Lipid $_{\text {crevice }}$ Figure 2) and between the scaffold and the transport domain of its neighboring subunit (Arkhipova et al., 2020; Wang and Boudker, 2020). Lipid moieties are also found in the spaces between the scaffold and transport domains (Akyuz et al., 2015; Arkhipova et al., 2020; Wang and Boudker, 2020). A notable one is inserted between the HP2a helix and the scaffold TM4a (Lipid ${ }_{\text {Out }}$ Figure 2). It is found at the same location for both the OFS and IFS Asp-bound GIt $t_{\mathrm{Ph}}$. HP2 opening in both OFS and IFS requires the displacement of this lipid. During the outwardto-inward transition, as HP2 slides past TM4a, the density corresponding to this lipid seems disordered in Glt $\mathrm{ph}_{\mathrm{ph}}$-iOFSAsp. Therefore, the lipid molecules at this site could potentially modulate gating and the translocation dynamics. In IFS where the transport domain leans away from the scaffold, like $\mathrm{Glt}_{\mathrm{ph}}{ }^{-}$ IFS-TFBTBOA, Glt $t_{\mathrm{Ph}}$-IFS-Asp R276S/M395R, and GIt $\mathrm{Ph}_{\mathrm{Ph}}$ IFS-Apo open, excess lipid-like densities are found in the crevice at the cytoplasmic side of the transport and the scaffold (Figure 4B).

There is also large overall lipid membrane deformation observed in both $\mathrm{Glt}_{\mathrm{Tk}}$ and $\mathrm{Glt}_{\mathrm{Ph}}$ nanodisc complexes (Arkhipova et al., 2020; Wang and Boudker, 2020). Protomers of the transporters in the OFS induce little membrane bending. In the IFS, in $\mathrm{Glt}_{\mathrm{Ph}}{ }^{-}$ IFS-Asp the lipid bilayer around protomers strongly bends down and in Glt $_{\mathrm{Ph}}$-IFS-TFBTBOA the membrane bends up as the transport domain leans away. Specific protein-lipid interactions may balance the large energetic penalty of such deformation (Zhou et al., 2019). It was reported that Glt $\mathrm{t}_{\mathrm{Ph}}$ transport activity increases in the presence of Phosphatidylethanolamine (PE), in comparison with Phosphatidylcholine (PC) only, perhaps due to either specific interactions between the protein and lipid headgroups, or by properties of the bilayer such as curvature and fluidity (Mcllwain et al., 2015). In EAAT2, cholesterol has been shown to be essential for its localization and function (Butchbach et al., 2004). Amphiphilic molecules like arachidonic acid have been shown to regulate glutamate transport activities based on EAAT subtypes (Zerangue et al., 1995; Fairman et al., 1998; Trotti et al., 1995; Tzingounis et al., 1998).

\section{CONCLUSION AND PERSPECTIVES}

In summary, the glutamate transporter structures solved by crystallography and Cryo-EM unveiled critical steps of the transport cycle (Figure 7). The transporter functions like an elevator, with the transport domain slides up and down, through intermediate states, relative to a "fixed barrier" formed by the scaffold domain (Drew and Boudker, 2016). Substrate and $\mathrm{Na}^{+}$ ions binding in both OFS and IFS are coupled to the opening of one single gate: HP2. Gating in OFS requires HP2 opening itself. In contrast, gating in IFS involves local restructure of HP2 coupled to a large transport domain swing-out (Figure 7). Still, many questions remain unanswered in the field. Singlemolecule transport assay has recently shown that individual Glt $t_{P h}$ transporters can function at rates varying by more than two orders of magnitude that persist for multiple turnovers (Ciftci et al., 2020). Mutations with enhanced Asp uptake rates show a shift of population to faster OFS/IFS dynamics and substrate-releasing rates (Huysmans et al., 2020). Therefore, uncharacterized conformational states might be responsible for distinguishing the subset of transporters with fast dynamics from those with slow dynamics. With the advance of Cryo-EM data acquisition and data processing, acquiring high-resolution structures of glutamate transporters in heterogeneous states is more likely to succeed than before. We also anticipate more structures of the EAATs coming out soon. They would answer crucial questions like the gating mechanism of EAATs in the IFS, the proton-coupling mechanism, and most importantly, how $\mathrm{K}^{+}$ ion binds to IFS of EAAT and reorient the transporter back to OFS. The structure bases of the uncoupled $\mathrm{Cl}$ - conductance in glutamate transporters could be studied as well, using $\mathrm{Glt}_{\mathrm{Ph}}$ as a model, or directly on EAATs. As current EAAT structures are all solved in detergent, further structural studies could be done in lipid environment, using the nanodisc system. Together with smFRET, single-molecule transport assay, HS-AFM, NMR and other tools available, the structure and function relationship of EAATs would be investigated in the future. As a clinical target of EAAT2 is to speed up the transporter to prevent neuronal damage caused by glutamate overflow, it would be highly intriguing to acquire EAAT structures in the presence of reported 
transport activators (Falcucci et al., 2019).

\section{ACKNOWLEDGEMENTS}

We thank Dr. Olga Boudker's laboratory at Weill Cornell Medicine for support of our research.

Original Submission: Nov 24, 2020

Revised Version Received: Dec 19, 2020

Accepted: Dec 20, 2020

\section{REFERENCES}

Akyuz, N., Altman, R.B., Blanchard, S.C., and Boudker, O. (2013). Transport dynamics in a glutamate transporter homologue. Nature $\mathbf{5 0 2}$, 114-118.

Akyuz, N., Georgieva, E.R., Zhou, Z., Stolzenberg, S., Cuendet, M.A., Khelashvili, G., Altman, R.B., Terry, D.S., Freed, J.H., Weinstein, H., Boudker, O., and Blanchard, S.C. (2015). Transport domain unlocking sets the uptake rate of an aspartate transporter. Nature 518, 68-73.

Arkhipova, V., Guskov, A., and Slotboom, D.J. (2020). Structural ensemble of a glutamate transporter homologue in lipid nanodisc environment. Nat Commun 11, 998.

Bastug, T., Heinzelmann, G., Kuyucak, S., Salim, M., Vandenberg, R.J., and Ryan, R.M. (2012). Position of the third $\mathrm{Na}+$ site in the aspartate transporter GItPh and the human glutamate transporter, EAAT1. PLoS One 7, e33058.

Bendahan, A., Armon, A., Madani, N., Kavanaugh, M.P., and Kanner, B.I. (2000). Arginine 447 plays a pivotal role in substrate interactions in a neuronal glutamate transporter. J Biol Chem 275, 37436-37442.

Boudker, O., Ryan, R.M., Yernool, D., Shimamoto, K., and Gouaux, E. (2007). Coupling substrate and ion binding to extracellular gate of a sodium-dependent aspartate transporter. Nature 445, 387-393.

Butchbach, M.E., Tian, G., Guo, H., and Lin, C.L. (2004). Association of excitatory amino acid transporters, especially EAAT2, with cholesterol-rich lipid raft microdomains: importance for excitatory amino acid transporter localization and function. J Biol Chem 279, 34388-34396.

Canul-Tec, J.C., Assal, R., Cirri, E., Legrand, P., Brier, S., Chamot-Rooke, J., and Reyes, N. (2017). Structure and allosteric inhibition of excitatory amino acid transporter 1. Nature 544, 446-451.

Ciftci, D., Huysmans, G.H.M., Wang, X., He, C., Terry, D., Zhou, Z., Fitzgerald, G., Blanchard, S.C., and Boudker, O. (2020). Single-molecule transport kinetics of a glutamate transporter homolog shows static disorder. Sci Adv 6, eaaz1949.

Conradt, M., and Stoffel, W. (1995). Functional analysis of the high affinity, $\mathrm{Na}(+)$-dependent glutamate transporter GLAST-1 by site-directed mutagenesis. J Biol Chem 270, 25207-25212.

Crisman, T.J., Qu, S., Kanner, B.I., and Forrest, L.R. (2009). Inward-facing conformation of glutamate transporters as revealed by their invertedtopology structural repeats. Proc Natl Acad Sci U S A 106, 20752-20757.

Danbolt, N.C. (2001). Glutamate uptake. Prog Neurobiol 65, 1-105.

Drew, D., and Boudker, O. (2016). Shared molecular mechanisms of membrane transporters. Annu Rev Biochem 85, 543-572.

Engelke, T., Jording, D., Kapp, D., and Pühler, A. (1989). Identification and sequence analysis of the Rhizobium meliloti dctA gene encoding the C4dicarboxylate carrier. J Bacteriol 171, 5551-5560.

Erkens, G.B., Hänelt, I., Goudsmits, J.M., Slotboom, D.J., and van Oijen, A.M. (2013). Unsynchronised subunit motion in single trimeric sodiumcoupled aspartate transporters. Nature 502, 119-123.
Fairman, W.A., Sonders, M.S., Murdoch, G.H., and Amara, S.G. (1998). Arachidonic acid elicits a substrate-gated proton current associated with the glutamate transporter EAAT4. Nat Neurosci 1, 105-113.

Fairman, W.A., Vandenberg, R.J., Arriza, J.L., Kavanaugh, M.P., and Amara, S.G. (1995). An excitatory amino-acid transporter with properties of a ligand-gated chloride channel. Nature 375, 599-603.

Falcucci, R.M., Wertz, R., Green, J.L., Meucci, O., Salvino, J., and Fontana, A.C.K. (2019). Novel positive allosteric modulators of glutamate transport have neuroprotective properties in an in vitro excitotoxic model. ACS Chem Neurosci 10, 3437-3453.

Focke, P.J., Moenne-Loccoz, P., and Larsson, H.P. (2011). Opposite movement of the external gate of a glutamate transporter homolog upon binding cotransported sodium compared with substrate. J Neurosci 31, 6255-6262.

Garaeva, A.A., Guskov, A., Slotboom, D.J., and Paulino, C. (2019). A onegate elevator mechanism for the human neutral amino acid transporter ASCT2. Nat Commun 10, 3427.

Garaeva, A.A., and Slotboom, D.J. (2020). Elevator-type mechanisms of membrane transport. Biochem Soc Trans 48, 1227-1241.

Georgieva, E.R., Borbat, P.P., Ginter, C., Freed, J.H., and Boudker, O. (2013). Conformational ensemble of the sodium-coupled aspartate transporter. Nat Struct Mol Biol 20, 215-221.

Groeneveld, M., and Slotboom, D.J. (2010). Na(+):aspartate coupling stoichiometry in the glutamate transporter homologue Glt(Ph). Biochemistry 49, 3511-3513.

Guskov, A., Jensen, S., Faustino, I., Marrink, S.J., and Slotboom, D.J. (2016). Coupled binding mechanism of three sodium ions and aspartate in the glutamate transporter homologue GltTk. Nat Commun 7, 13420.

Hänelt, I., Wunnicke, D., Bordignon, E., Steinhoff, H.J., and Slotboom, D.J. (2013). Conformational heterogeneity of the aspartate transporter Glt(Ph). Nat Struct Mol Biol 20, 210-214.

Huang, Y., Wang, X., Lv, G., Razavi, A.M., Huysmans, G.H.M., Weinstein, H., Bracken, C., Eliezer, D., and Boudker, O. (2020). Use of paramagnetic $19 \mathrm{~F}$ NMR to monitor domain movement in a glutamate transporter homolog. Nat Chem Biol 16, 1006-1012.

Huysmans, G.H.M., Ciftci, D., Wang, X., Blanchard, S.C., and Boudker, O. (2020). The high-energy transition state of the glutamate transporter homologue GItPh. EMBO J, e105415

Jensen, S., Guskov, A., Rempel, S., Hänelt, I., and Slotboom, D.J. (2013). Crystal structure of a substrate-free aspartate transporter. Nat Struct Mol Biol 20, 1224-1226.

Koch, H.P., and Larsson, H.P. (2005). Small-scale molecular motions accomplish glutamate uptake in human glutamate transporters. $J$ Neurosci 25, $1730-1736$.

Matin, T.R., Heath, G.R., Huysmans, G.H.M., Boudker, O., and Scheuring, S. (2020). Millisecond dynamics of an unlabeled amino acid transporter. Nat Commun 11, 5016.

Mcllwain, B.C., Vandenberg, R.J., and Ryan, R.M. (2015). Transport rates of a glutamate transporter homologue are influenced by the lipid bilayer. $J$ Biol Chem 290, 9780-9788.

Menaker, D., Bendahan, A., and Kanner, B.I. (2006). The substrate specificity of a neuronal glutamate transporter is determined by the nature of the coupling ion. J Neurochem 99, 20-28.

Oh, S., and Boudker, O. (2018). Kinetic mechanism of coupled binding in sodium-aspartate symporter GltPh. Elife 7, e37291.

Reyes, N., Ginter, C., and Boudker, O. (2009). Transport mechanism of a bacterial homologue of glutamate transporters. Nature 462, 880-885.

Reyes, N., Oh, S., and Boudker, O. (2013). Binding thermodynamics of a glutamate transporter homolog. Nat Struct Mol Biol 20, 634-640. 
Ruan, Y., Miyagi, A., Wang, X., Chami, M., Boudker, O., and Scheuring, S. (2017). Direct visualization of glutamate transporter elevator mechanism by high-speed AFM. Proc Natl Acad Sci U S A 114, 1584-1588.

Ryan, R.M., and Mindell, J.A. (2007). The uncoupled chloride conductance of a bacterial glutamate transporter homolog. Nat Struct Mol Biol 14, 365371.

Ryan, R.M., Mitrovic, A.D., and Vandenberg, R.J. (2004). The chloride permeation pathway of a glutamate transporter and its proximity to the glutamate translocation pathway. J Biol Chem 279, 20742-20751.

Scopelliti, A.J., Font, J., Vandenberg, R.J., Boudker, O., and Ryan, R.M. (2018). Structural characterisation reveals insights into substrate recognition by the glutamine transporter ASCT2/SLC1A5. Nat Commun $\mathbf{9}$, 38.

Seal, R.P., Leighton, B.H., and Amara, S.G. (2000). A model for the topology of excitatory amino acid transporters determined by the extracellular accessibility of substituted cysteines. Neuron 25, 695-706.

Silverstein, N., Crisman, T.J., Forrest, L.R., and Kanner, B.I. (2013). Cysteine scanning mutagenesis of transmembrane helix 3 of a brain glutamate transporter reveals two conformationally sensitive positions. $J$ Biol Chem 288, 964-973.

Slotboom, D.J., Konings, W.N., and Lolkema, J.S. (2001). Glutamate transporters combine transporter- and channel-like features. Trends Biochem Sci 26, 534-539.

Tao, Z., Rosental, N., Kanner, B.I., Gameiro, A., Mwaura, J., and Grewer, C. (2010). Mechanism of cation binding to the glutamate transporter EAAC1 probed with mutation of the conserved amino acid residue Thr101. J Biol Chem 285, 17725-17733.

Trotti, D., Volterra, A., Lehre, K.P., Rossi, D., Gjesdal, O., Racagni, G., and Danbolt, N.C. (1995). Arachidonic acid inhibits a purified and reconstituted glutamate transporter directly from the water phase and not via the phospholipid membrane. J Biol Chem 270, 9890-9895.

Tzingounis, A.V., Lin, C.L., Rothstein, J.D., and Kavanaugh, M.P. (1998). Arachidonic acid activates a proton current in the rat glutamate transporter
EAAT4. J Biol Chem 273, 17315-17317.

Verdon, G., and Boudker, O. (2012). Crystal structure of an asymmetric trimer of a bacterial glutamate transporter homolog. Nat Struct Mol Biol 19, 355-357.

Verdon, G., Oh, S., Serio, R.N., and Boudker, O. (2014). Coupled ion binding and structural transitions along the transport cycle of glutamate transporters. Elife 3, e02283.

Veruki, M.L., Mørkve, S.H., and Hartveit, E. (2006). Activation of a presynaptic glutamate transporter regulates synaptic transmission through electrical signaling. Nat Neurosci 9, 1388-1396.

Wang, X., and Boudker, O. (2020). Large domain movements through the lipid bilayer mediate substrate release and inhibition of glutamate transporters. Elife 9, e58417.

Yernool, D., Boudker, O., Jin, Y., and Gouaux, E. (2004). Structure of a glutamate transporter homologue from Pyrococcus horikoshii. Nature 431, 811-818.

Yurgel, S.N., and Kahn, M.L. (2005). Sinorhizobium meliloti dctA mutants with partial ability to transport dicarboxylic acids. J Bacteriol 187, 11611172.

Zarbiv, R., Grunewald, M., Kavanaugh, M.P., and Kanner, B.I. (1998). Cysteine scanning of the surroundings of an alkali-ion binding site of the glutamate transporter GLT-1 reveals a conformationally sensitive residue. J Biol Chem 273, 14231-14237.

Zerangue, N., Arriza, J.L., Amara, S.G., and Kavanaugh, M.P. (1995). Differential modulation of human glutamate transporter subtypes by arachidonic acid. J Biol Chem 270, 6433-6435.

Zerangue, N., and Kavanaugh, M.P. (1996). Flux coupling in a neuronal glutamate transporter. Nature 383, 634-637.

Zhou, W., Fiorin, G., Anselmi, C., Karimi-Varzaneh, H.A., Poblete, H., Forrest, L.R., and Faraldo-Gómez, J.D. (2019). Large-scale statedependent membrane remodeling by a transporter protein. Elife $\mathbf{8}$, e50576. 